\title{
Gram-negative bacteremia and asplenia in a well 15-year-old girl
}

\author{
Mindy CW Lam MSc${ }^{1}$, Robert Verity $\mathrm{MD}^{2}$, Gregory J Tyrrell $\mathrm{PhD}^{3}$, \\ Robin Arent $M D^{4}$, Jana Nigrin $M D^{2}$, Sarah ED Forgie $M D^{1,4}$
}

A 15-year-old girl who had undergone a splenectomy at seven years of age for idiopathic thrombocytopenic purpura presented with a two-day history of fever, myalgia and headache. On examination, she was afebrile, did not appear toxic and no focus of infection was found. Her complete blood cell count showed a white blood cell count of $14 \times 10^{9} / \mathrm{L}$ with 91\% neutrophils, 4\% monocytes and 5\% lymphocytes; hemoglobin level of $145 \mathrm{~g} / \mathrm{L}$ and platelet count of $372 \times 10^{9} / \mathrm{L}$. Her serum electrolyte and creatinine levels were normal. Urine and blood cultures were obtained, and she was sent home without antibiotic therapy.

The urine culture grew mixed organisms suggestive of contamination, but Gram-negative bacilli grew in the aerobic blood culture vial (BACTEC 9240 Peds plus blood culture bottle, BD Diagnostics Inc, USA) after $45 \mathrm{~h}$ of incubation (Figure 1). The girl was, therefore, referred to the pediatric emergency department. Her fever, headache and myalgia had resolved. Physical examination again revealed a nontoxic female in no distress. Her temperature was $36.3^{\circ} \mathrm{C}$, heart rate was 70 beats/min, respiratory rate was 16 breaths $/ \mathrm{min}$, blood pressure was $90 / 60 \mathrm{mmHg}$ and oxygen saturation was $98 \%$ in room air. Aside from an abdominal scar, the physical examination was normal. Laboratory studies revealed a white blood cell count of $10.5 \times 10^{9} / \mathrm{L}$ with $51 \%$ neutrophils, $4 \%$ band forms, $7 \%$ monocytes and 38\% lymphocytes; hemoglobin level of $133 \mathrm{~g} / \mathrm{L}$ and platelet count of $167 \times 10^{9} / \mathrm{L}$. Red blood cell morphology was notable for $1+$ Howell-Jolly bodies. C-reactive protein level was $1.1 \mathrm{~g} / \mathrm{L}$ and erythrocyte sedimentation rate was $1 \mathrm{~mm} / \mathrm{h}$. Blood cultures were repeated. Because no obvious focus of infection was found and the patient appeared well, she was given a dose of intravenous ceftriaxone and was discharged to be reassessed in $24 \mathrm{~h}$.

On the day the original blood culture vial grew Gramnegative bacilli, it was subcultured onto $5 \%$ sheep blood agar in $\mathrm{CO}_{2}$, chocolate agar in $\mathrm{CO}_{2}$, MacConkey agar in ambient air and $\mathrm{CDC}$ anaerobic blood agar under anaerobic conditions. Within $24 \mathrm{~h}$, all agar incubated aerobically showed growth of Gram-negative bacilli, but the anaerobic media displayed no growth. The organism was not identified using the Gramnegative identification card on the VITEK 2 (bioMerieux



Figure 1) Gram-negative bacilli on Gram stain from the blood culture. Note single organisms throughout and chains in the centre and bottom left. Photo courtesy of Dr Nigrin

Canada Inc). A presumptive identification of Acinetobacter species was made two days later because of the biochemical characteristics (negative oxidase, indole and urease tests, positive catalase test and asaccharolytic profile) (1). Due to the atypical Gram stain appearance (smaller coccobacilli rather than larger coccobacilli and/or diplococci, typical of Acinetobacter species), the isolate was referred to the Alberta Provincial Laboratory for Public Health for further identification. The antimicrobial susceptibility was performed using an AST-GN10 card on the VITEK 2. The antimicrobial susceptibility results showed the following minimum inhibitory concentrations - ceftazidime $2 \mathrm{mg} / \mathrm{L}$ or lower, imipenem $4 \mathrm{mg} / \mathrm{L}$ or lower, piperacillin $8 \mathrm{mg} / \mathrm{L}$ or lower, trimethoprim/sulfamethoxizole $2 / 38 \mathrm{mg} / \mathrm{L}$ or lower, tobramycin $1 \mathrm{mg} / \mathrm{L}$ or lower and ciprofloxacin $1 \mathrm{mg} / \mathrm{L}$ or lower.

The patient was treated with a 14-day course of ciprofloxacin following the single dose of ceftriaxone; she remained asymptomatic. Two sets of blood cultures drawn before, and on day 4 of antibiotic therapy were negative. What organism was ultimately identified?

${ }^{1}$ Faculty of Medicine and Dentistry, University of Alberta; ${ }^{2}$ Microbiology, DynaLIFE ${ }_{D x}$, Diagnostic Laboratory Services; ${ }^{3}$ Division of Medical

Microbiology, Department of Laboratory Medicine and Pathology, University of Alberta; ${ }^{4}$ Department of Pediatrics and Stollery Children's

Hospital, Edmonton, Alberta

Correspondence: Dr Sarah Forgie, 8224A, Aberhart Centre, Edmonton, Alberta T6G 2J3. Telephone 780-407-1680, fax 780-407-7136, e-mail sarahforgie@cha.ab.ca

Received and accepted for publication January 28, 2008 


\section{DIAGNOSIS}

The bacterial isolate was a small Gram-negative coccobacillus that could not be definitively identified by biochemical analysis at the Provincial Laboratory for Public Health. As per their current algorithm for identifying unusual, small Gramnegative coccobacilli, they performed cellular fatty acid analysis. The organism was identified as Bordetella holmesii using the results from both the cellular fatty acid profile and the biochemical analysis.

\section{DISCUSSION}

To our knowledge, this is the first case of $B$ holmesii bacteremia in northern Alberta. This organism was first isolated in 1983 and formally named in 1995 (2). Although the epidemiology is not well defined, $B$ holmesii has been described as a cause of bacteremia, endocarditis and respiratory illness. Bacteremia has been described in immunocompromised patients with diabetes, lymphoma, long-term steroid therapy, solid organ transplant or AIDS (2-8). In the largest case series (3) of 30 patients with B holmesii bacteremia, $85 \%$ were functionally or anatomically asplenic. Contamination of blood cultures with B holmesii is yet to be described, and seems unlikely in our case because asplenia is a well-recognized risk factor for B holmesii bacteremia. There have been two case reports $(3,9)$ of $B$ holmesii endocarditis complicating bacteremia. With regard to respiratory illness, B holmesii has been described as a cause of a pertussis-like syndrome with cough (100\%), whoop (9\%), post-tussive vomiting (26\%) and coughing paroxysms (61\%), noted in a case series $(4,5)$ of 33 patients.

As in our case, the illness associated with B holmesii bacteremia is usually mild and nonspecific, with symptoms including headache $(50 \%)$, chills (38\%), vomiting (38\%), cough $(27 \%)$, a median temperature of $38.3^{\circ} \mathrm{C}$ at presentation and a median white blood cell count of $13.7 \times 10^{9} / \mathrm{L}(10)$. However, hospital admission is common in the face of Gramnegative bacteremia in an immunocompromised host. To date, there have been no reports of death associated with B holmesii infection $(3,9)$.

Laboratory identification of the organism may pose some difficulty. B holmesii is a slow-growing, Gram-negative, oxidasenegative, urease-negative and asaccharolytic organism $(3,10)$. Unlike other Bordetella species, B holmesii has no oxidase activity, and unlike Acinetobacter species, it produces a brown soluble pigment $(2,10,11)$. Our patient's bacteremia had resolved before administration of antimicrobials, and it seemed likely that she would have made an uneventful recovery even without antibiotics. It is, therefore, possible that many cases of $B$ holmesii bacteremia are not recognized. In theory, the treatment of B holmesii should be challenging given the potential for misidentification, the lack of established susceptibility breakpoints and the limited data on the efficacy of antimicrobials, but treatment failures are yet to be described despite the use of a wide variety of antimicrobials including aminoglycosides, macrolides and cephalosporins.

ACKNOWLEDGEMENT: The authors thank Dr Joan Robinson for her help in editing the present manuscript.

\section{REFERENCES}

1. Schreckenberger PC, Daneshvar MI, Weyant RS, Hollis DG. Acinetobacter, Achromobacter, Chryseobacterium, Moraxella, and other nonfermentative Gram-negative rods. In: Murray PR, Baron EJ, Jorgensen JH, Pfaller MA, Yolken RH, eds. Manual of Clinical Microbiology, 8th edn. Washington: American Society for Microbiology Press 2003;749-79.

2. Weyant RS, Hollis DG, Weaver RE, et al. Bordetella holmesii sp. nov., a new gram-negative species associated with septicemia. J Clin Microbiol 1995;33:1-7.

3. Shepard CW, Daneshvar MI, Kaiser RM, et al. Bordetella holmesii bacteremia: A newly recognized clinical entity among asplenic patients. Clin Infect Dis 2004;38:799-804.

4. Yih WK, Silva EA, Ida J, Harrington N, Lett SM, George H. Bordetella holmesii-like organisms isolated from Massachusetts patients with pertussis-like symptoms. Emerg Infect Dis 1999;5:441-3.

5. Knorr L, Fox JD, Tilley PA, Ahmed-Bentley J. Evaluation of real-time PCR for diagnosis of Bordetella pertussis infection. BMC Infect Dis 2006; 6:62-74.

6. Morris JT, Myers M. Bacteremia due to Bordetella holmesii. Clin Infect Dis 1998;27:912-3.

7. Lindquist SW, Weber DJ, Mangum ME, Hollis DG, Jordan J. Bordetella holmesii sepsis in an asplenic adolescent. Pediatr Infect Dis J 1995;14:813-5.

8. Russell FM, Davis JM, Whipp MJ, et al. Severe Bordetella holmesii infection in a previously healthy adolescent confirmed by gene sequence analysis. Clin Infect Dis 2001;33:129-30.

9. Tang Y, Hopkins MK, Kolbert CP, Hartley PA, Severance PJ, Persing DH. Bordetella-holmesii-like organisms associated with septicemia, endocarditis and respiratory failure. Clin Infect Dis 1998;26:389-92.

10. Bergogne-Berezin E, Towner KJ. Acinetobacter spp. as nosocomial pathogens: Microbiological, clinical, and epidemiological features. Clin Microbiol Rev 1996;9:148-65.

11. Hollis DG, Moss CW, Daneshvar MI, et al. Characterization of Centers for Disease Control group NO-1, a fastidious, nonoxidative, gram-negative organism associated with dog and cat bites. J Clin Microbiol 1993;31:746-8. 


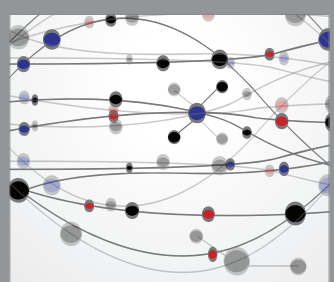

The Scientific World Journal
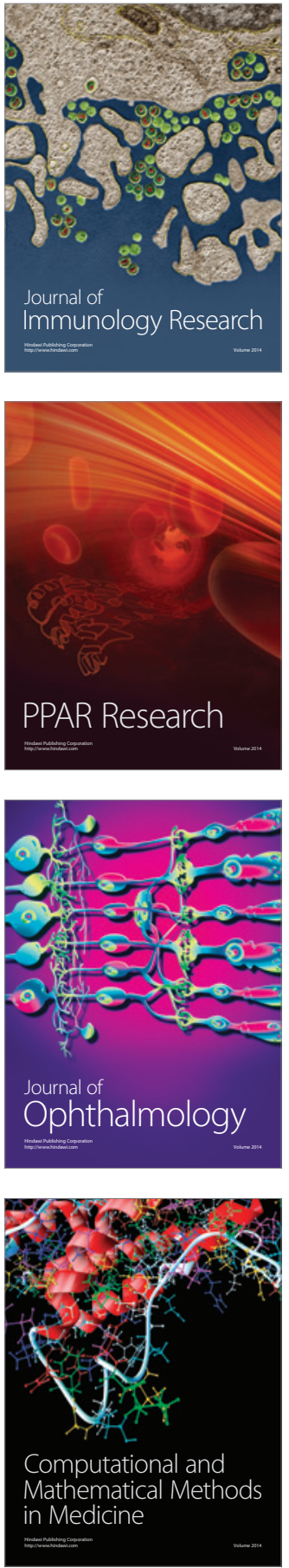

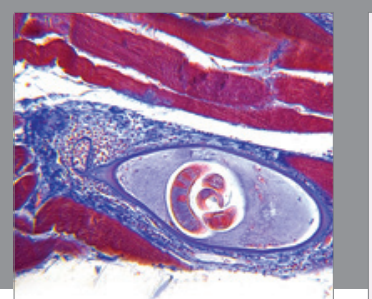

Gastroenterology Research and Practice

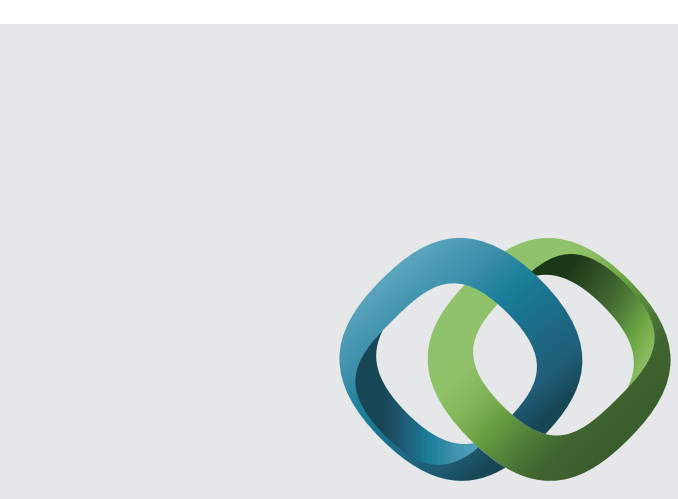

\section{Hindawi}

Submit your manuscripts at

http://www.hindawi.com
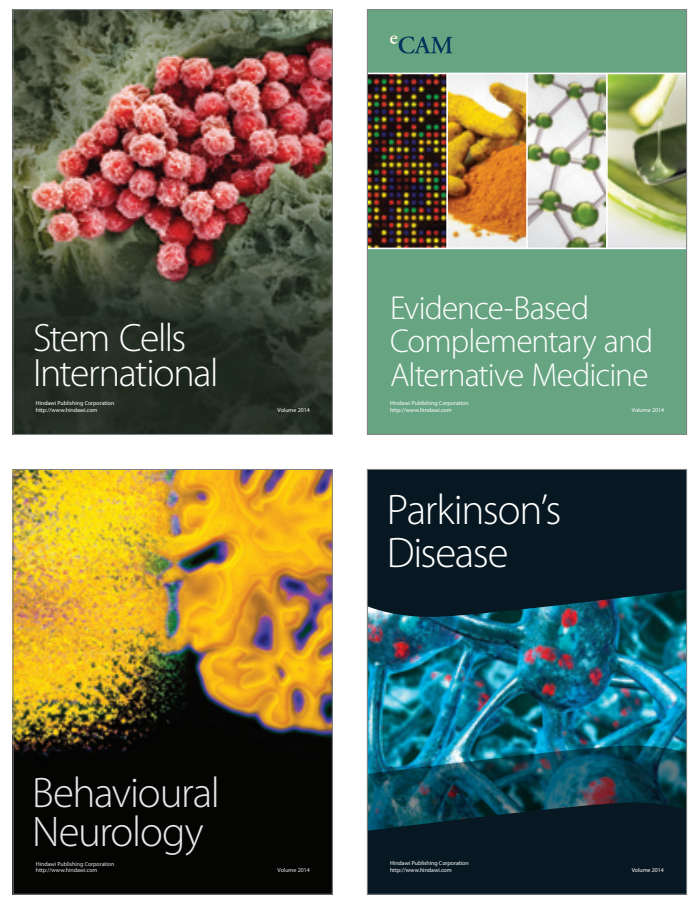
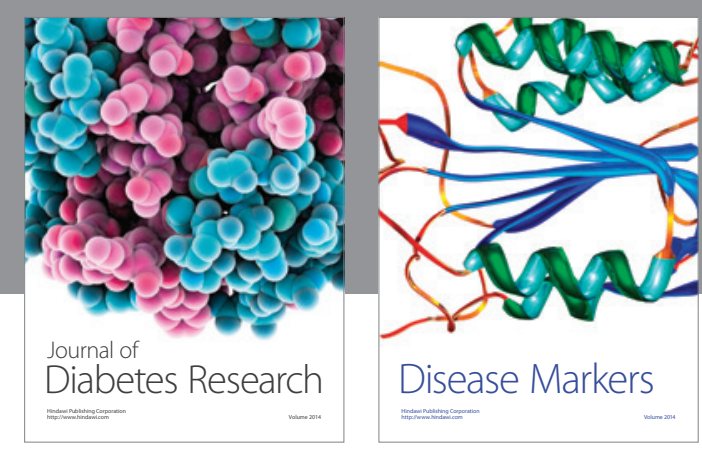

Disease Markers
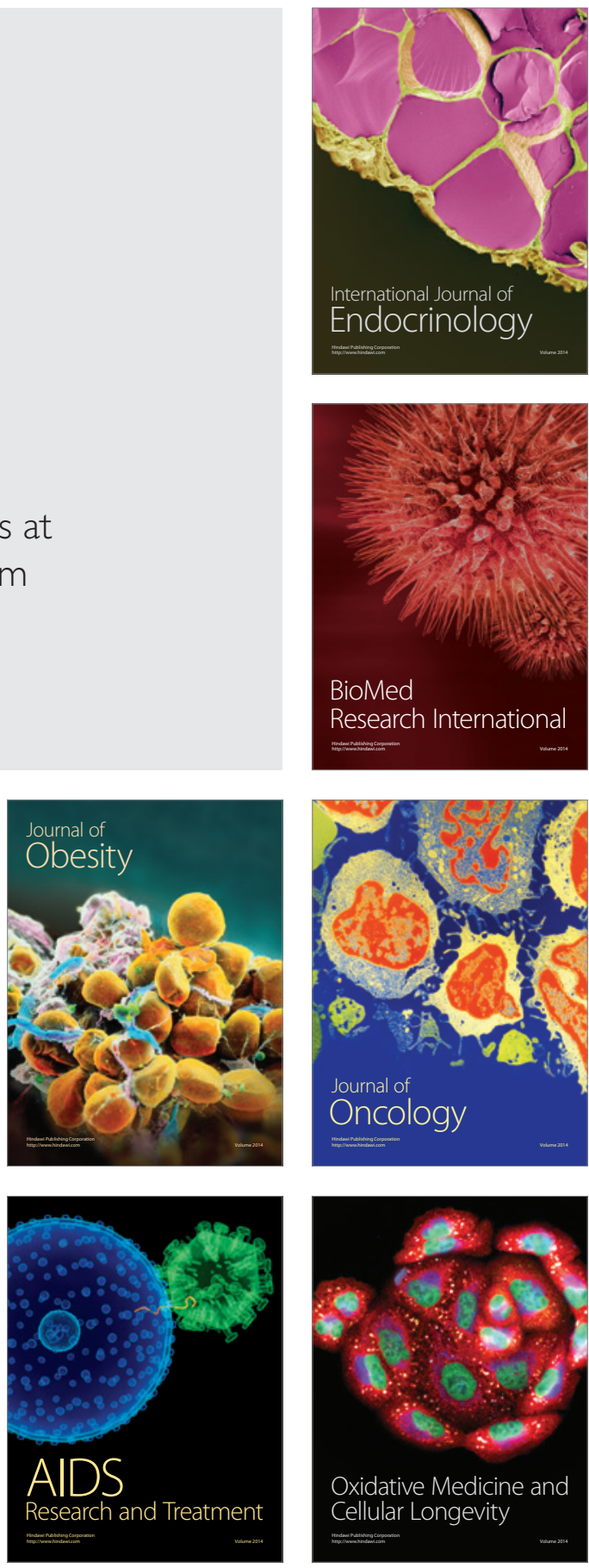